\title{
THE GENERALIZED METHOD OF EXHAUSTION
}

\author{
ANTHONY A. RUFFA
}

Received 22 January 2001 and in revised form 15 June 2001

\begin{abstract}
The method of exhaustion is generalized to a simple integration formula that is valid for the Riemann integrable functions. Both a geometric approach (following the usual procedure for the method of exhaustion) and an independent algebraic generalization approach are provided. Applications provided as examples include use of the formula to generate new series for common functions as well as computing the group velocity distribution resulting from waves diffracted from an aperture.
\end{abstract}

2000 Mathematics Subject Classification: 26A06, $26 B 15$.

1. Introduction. The method of exhaustion was used by the ancient Greeks to integrate a limited number of functions before the development of calculus [6]. It will be shown here that this ancient method can be generalized to a simple formula to integrate all functions that are Riemann integrable over the interval in question. The formula is

$$
\int_{a}^{b} f(x) d x=(b-a) \sum_{n=1}^{\infty} \sum_{m=1}^{2^{n}-1}(-1)^{m+1} 2^{-n} f\left(a+\frac{m(b-a)}{2^{n}}\right) .
$$

The generalization is simple and geometric in nature, directly following the procedure originally used for the method of exhaustion. An independent algebraic generalization approach is also provided for the special case for which $a=0$.

2. Geometric approach. The generalization approach uses the successive approximation method historically used to obtain the exact area under the simple curves previously integrated by the method of exhaustion. Consider an arbitrary function $f(x)$ that is piecewise continuous on $[a, b]$. The procedure to find the area under $f(x)$ on $[a, b]$ involves successive approximations with triangles that first intersect $f(x)$ at the midpoint, $x=a+(b-a) / 2$, and then at the new midpoints, $x=a+(b-a) / 4$, $x=a+3(b-a) / 4$, and so on. The first such approximation is shown in Figure 2.1. Its area is

$$
A_{1}=\frac{1}{2}(b-a) f\left(a+\frac{b-a}{2}\right) .
$$

The second such approximation adds two new triangles (Figure 2.2), each sharing two vertices with the original triangle, and each bisecting one of the remaining arcs of $f(x)$ at its third vertex. The two triangles have the area

$$
A_{2}=\frac{1}{4}(b-a)\left\{f\left(a+\frac{b-a}{4}\right)-f\left(a+\frac{b-a}{2}\right)+f\left(a+\frac{3(b-a)}{4}\right)\right\} .
$$




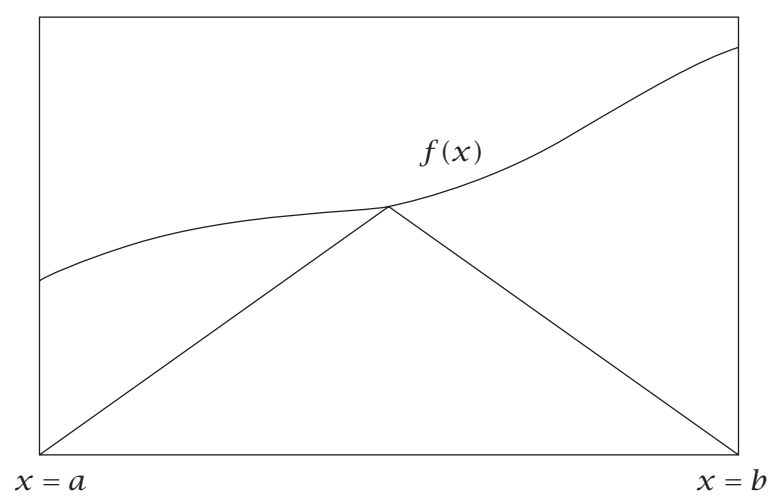

FIGURE 2.1. First approximation to the area under $f(x)$.

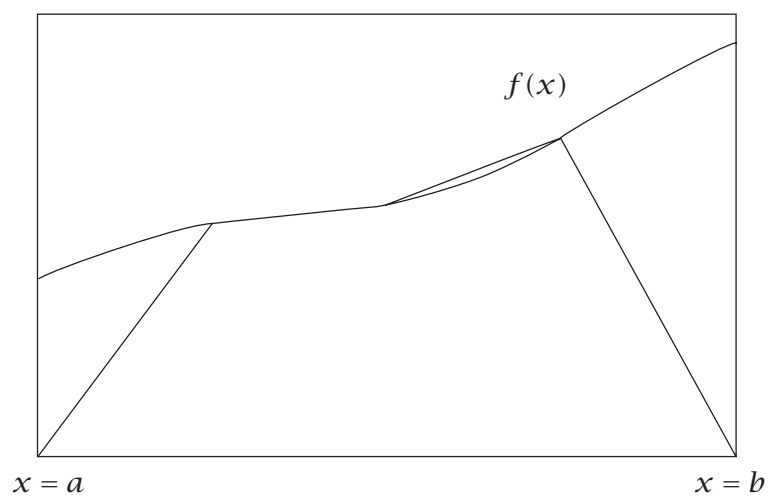

FigURE 2.2. Second approximation to the area under $f(x)$.

A third such approximation (Figure 2.3) adds the area

$$
\begin{aligned}
A_{3}=\frac{1}{8}(b-a)\{ & f\left(a+\frac{b-a}{8}\right)-f\left(a+\frac{b-a}{4}\right)+f\left(a+\frac{3(b-a)}{8}\right)-f\left(a+\frac{b-a}{2}\right) \\
& \left.+f\left(a+\frac{5(b-a)}{8}\right)-f\left(a+\frac{3(b-a)}{4}\right)+f\left(a+\frac{7(b-a)}{8}\right)\right\} .
\end{aligned}
$$

Each new iteration $n$ leads to $\left(2^{n}-1\right)$ new triangles adding area $A_{n}$ to further refine the area estimate. This procedure is continued indefinitely to exhaust the remaining area, leading to the formula

$$
\int_{a}^{b} f(x) d x=\sum_{n=1}^{\infty} A_{n}=(b-a) \sum_{n=1}^{\infty} \sum_{m=1}^{2^{n}-1}(-1)^{m+1} 2^{-n} f\left(a+\frac{m(b-a)}{2^{n}}\right) .
$$




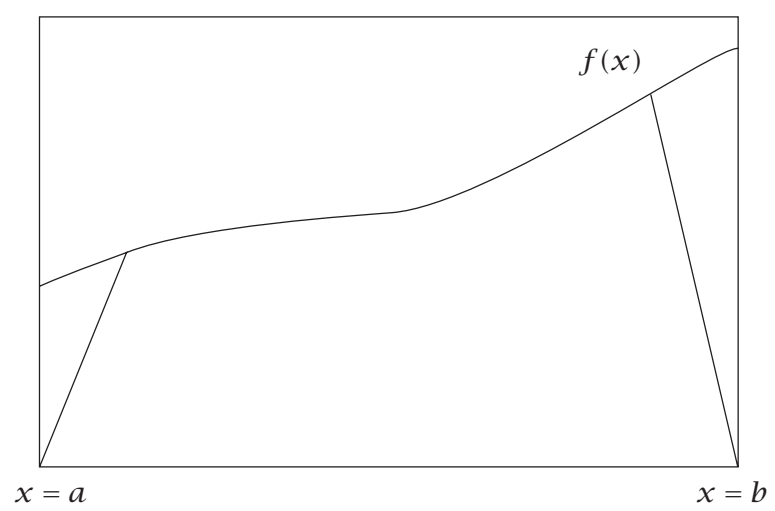

FIGURE 2.3. Third approximation to the area under $f(x)$.

The special case for (2.4) when $a=0$ is as follows:

$$
\int_{0}^{b} f(x) d x=b \sum_{n=1}^{\infty} \sum_{m=1}^{2^{n}-1}(-1)^{m+1} 2^{-n} f\left(\frac{m b}{2^{n}}\right) .
$$

The method of exhaustion will converge to the value of the integral at least as fast as a geometric series, because when each new triangle is small enough so that the local curvature between intersection points on $f(x)$ is slowly varying, it will exhaust more than $1 / 2$ of the remaining area left from the previous approximation.

There are an infinite number of possible variations on this procedure. For example, each new iteration may involve a different number of triangles to exhaust the remaining area, or each new triangle could intersect the function at a location other than the midpoint.

3. Algebraic approach. An independent approach leading to (2.5) can be obtained starting with the identity [5]

$$
\frac{\sin a}{a}=\cos ^{2}\left(\frac{a}{2}\right)+\sum_{n=1}^{\infty} \sin ^{2}\left(\frac{a}{2^{n+1}}\right) \prod_{m=1}^{n} \cos \left(\frac{a}{2^{m}}\right) .
$$

This expression can be rewritten as follows:

$$
\frac{\sin a}{a}=\sum_{n=1}^{\infty} \sum_{m=1}^{2^{n}-1}(-1)^{m+1} 2^{-n} \cos \left(\frac{m a}{2^{n}}\right)
$$

or

$$
\frac{\sin b a}{a}=b \sum_{n=1}^{\infty} \sum_{m=1}^{2^{n}-1}(-1)^{m+1} 2^{-n} \cos \left(\frac{m b a}{2^{n}}\right) .
$$


Since

$$
\frac{\sin b a}{a}=\frac{1}{2} \int_{-b}^{b} e^{-i a x} d x
$$

it follows that

$$
\sum_{k=0}^{\infty} i^{k} \gamma_{k} \frac{d^{k}}{d a^{k}}\left[\frac{\sin (b a)}{a}\right]=\frac{1}{2} \int_{-b}^{b} \sum_{k=0}^{\infty} \gamma_{k} x^{k} e^{-i a x} d x
$$

Defining

$$
G(x)=\sum_{k=0}^{\infty} \gamma_{k} x^{k}
$$

and noting that

$$
\begin{aligned}
\sum_{k=0}^{\infty} i^{k} \gamma_{k} \frac{d^{k}}{d a^{k}}\left[\frac{\sin (b a)}{a}\right]= & b \sum_{n=1}^{\infty} \sum_{m=1}^{2^{n}-1}(-1)^{m+1} 2^{-n} \sum_{k=0}^{\infty} \gamma_{2 k}\left(\frac{m b}{2^{n}}\right)^{2 k} \cos \left(\frac{m b a}{2^{n}}\right) \\
& -i b \sum_{n=1}^{\infty} \sum_{m=1}^{2^{n}-1}(-1)^{m+1} 2^{-n} \sum_{k=0}^{\infty} \gamma_{2 k+1}\left(\frac{m b}{2^{n}}\right)^{2 k+1} \sin \left(\frac{m b a}{2^{n}}\right),
\end{aligned}
$$

it follows that

$$
\begin{aligned}
\int_{-b}^{b} G(x) e^{-i a x} d x= & b \sum_{n=1}^{\infty} \sum_{m=1}^{2^{n}-1}(-1)^{m+1} 2^{-n}\left[G\left(\frac{m b}{2^{n}}\right)+G\left(-\frac{m b}{2^{n}}\right)\right] \cos \left(\frac{m b a}{2^{n}}\right) \\
& -i b \sum_{n=1}^{\infty} \sum_{m=1}^{2^{n}-1}(-1)^{m+1} 2^{-n}\left[G\left(\frac{m b}{2^{n}}\right)-G\left(-\frac{m b}{2^{n}}\right)\right] \sin \left(\frac{m b a}{2^{n}}\right) .
\end{aligned}
$$

Setting

$$
G(x)=H(x) e^{i a x}
$$

leads to

$$
\int_{-b}^{b} H(x) d x=b \sum_{n=1}^{\infty} \sum_{m=1}^{2^{n}-1}(-1)^{m+1} 2^{-n}\left[H\left(\frac{m b}{2^{n}}\right)+H\left(-\frac{m b}{2^{n}}\right)\right] .
$$

Note that $H(x)$ can be in the form of a Fourier series which sums to the following:

$$
H(x)= \begin{cases}0, & -b \leq x<0 \\ f(x), & 0 \leq x<b\end{cases}
$$

Here $f(x)$ is piecewise continuous on $[0, b]$. This leads to

$$
\int_{0}^{b} f(x) d x=b \sum_{n=1}^{\infty} \sum_{m=1}^{2^{n}-1}(-1)^{m+1} 2^{-n} f\left(\frac{m b}{2^{n}}\right) .
$$


4. Equivalence to Riemann sums. It can be shown that (1.1) is valid for the Riemann integrable functions [4]. Consider the partial sums

$$
\begin{aligned}
A_{1}=\frac{1}{2}(b-a) f\left(a+\frac{b-a}{2}\right) ; \\
A_{1}+A_{2}=\frac{1}{4}(b-a)\left\{f\left(a+\frac{b-a}{4}\right)+f\left(a+\frac{b-a}{2}\right)+f\left(a+\frac{3(b-a)}{4}\right)\right\} ; \\
A_{1}+A_{2}+A_{3}=\frac{1}{8}(b-a)\left\{f\left(a+\frac{b-a}{8}\right)+f\left(a+\frac{b-a}{4}\right)+f\left(a+\frac{3(b-a)}{8}\right)\right. \\
+f\left(a+\frac{b-a}{2}\right)+f\left(a+\frac{5(b-a)}{8}\right)+f\left(a+\frac{3(b-a)}{4}\right) \\
\left.+f\left(a+\frac{7(b-a)}{8}\right)\right\} .
\end{aligned}
$$

This can be generalized as follows:

$$
\sum_{n=1}^{N} A_{n}=\frac{(b-a)}{2^{N}} \sum_{m=1}^{2^{N}-1} f\left(a+\frac{m(b-a)}{2^{N}}\right) .
$$

This is precisely the Riemann sum of order $2^{N}$ for $f(x)$, except for the two missing terms $f(a)$ and $f(b)$, which can be neglected in the limit $N \rightarrow \infty$.

5. Applications. The most immediate applications are series expressions for common functions that might otherwise be very difficult to derive. Some examples are presented as follows:

$$
\begin{gathered}
\sin x=x \sum_{n=1}^{\infty} \sum_{m=1}^{2^{n}-1}(-1)^{m+1} 2^{-n} \cos \left(\frac{m x}{2^{n}}\right) ; \\
\cos x=1-x \sum_{n=1}^{\infty} \sum_{m=1}^{2^{n}-1}(-1)^{m+1} 2^{-n} \sin \left(\frac{m x}{2^{n}}\right) ; \\
\int_{0}^{b} \frac{\sin a x}{x} d x=\sum_{n=1}^{\infty} \sum_{m=1}^{2^{n}-1} \frac{(-1)^{m+1}}{m} \sin \left(\frac{m b a}{2^{n}}\right) ; \\
e^{x}=1+x \sum_{n=1}^{\infty} \sum_{m=1}^{2^{n}-1}(-1)^{m+1} 2^{-n} e^{m x / 2^{n}} ; \\
\int^{-a x^{2}} d x=b \sum_{n=1}^{\infty} \sum_{m=1}^{2^{n}-1}(-1)^{m+1} 2^{-n} e^{-a(m b)^{2} / 4^{n}} ; \\
\ln x=\sum_{n=1}^{\infty} \sum_{m=1}^{1} \frac{(-1)^{m+1}(x-1)}{2^{n}+m(x-1)} \quad(0<x<\infty) ; \\
d x=\sum_{n=1}^{\infty} \sum_{m=1}^{2^{n}-1}(-1)^{m+1} 2^{-n}\left[\ln \left(\frac{2^{n}}{m}\right)\right]^{p} \quad(0 \leq p<\infty) .
\end{gathered}
$$

In addition to the above expressions, this method can lead to new insights into certain physical problems. One example involves the diffraction of waves though a 
two-dimensional aperture in an infinite screen. This problem has been solved exactly in integral form $[1,2,3]$ so that the field at every point depends on the Fourier transform $F\left(k_{x}, k_{y}\right)$ of the aperture at $z=0$

$$
\phi(x, y, z, t)=\frac{e^{-i \omega_{0} t}}{2 \pi} \int_{-\infty}^{\infty} \int_{-\infty}^{\infty} F\left(k_{x}, k_{y}\right) e^{-i k_{x} x} e^{-i k_{y} y} e^{i z \sqrt{k^{2}-k_{x}^{2}-k_{y}^{2}}} d k_{x} d k_{y} .
$$

Note that (5.2) satisfies the Helmholtz equation,

$$
\nabla^{2} \phi+k^{2} \phi=0
$$

everywhere $\left(k=\omega_{0} / c\right)$ as well as the boundary conditions on the screen.

Under suitable conditions, the extension of (2.4) to convergent improper integrals can be made as follows, that is,

$$
\int_{0}^{\infty} f(x) d x=b \sum_{n=1}^{\infty} \sum_{m=1}^{2^{n}-1} \sum_{p=0}^{\infty}(-1)^{m+1} 2^{-n} f\left(p b+\frac{m b}{2^{n}}\right) .
$$

Equation (5.4) is a result of expressing the integral as a series of definite integrals on $[p b,(p+1) b]$. The result is valid when each integral

$$
\int_{p b}^{(p+1) b} f(x) d x
$$

exists and when

$$
\int_{0}^{B} f(x) d x
$$

tends to a finite limit $L$ as $B \rightarrow \infty$.

With regard to (5.2), however, it can be noted that propagating waves only occur when the transverse wavenumber $\left(k_{x}^{2}+k_{y}^{2}\right)^{1 / 2}$ is lower than the cutoff wavenumber, so in the far field the integral can be evaluated to $\pm k$ with good accuracy as follows (assuming that $F\left(k_{x}, k_{y}\right)$ is an even function with respect to both $k_{x}$ and $k_{y}$ ):

$$
\begin{aligned}
\phi(x, y, z, t) \simeq & \frac{2 k^{2}}{\pi} e^{-i \omega_{0} t} \sum_{n=1}^{\infty} \sum_{m=1}^{2^{n}-1} \sum_{p=1}^{\infty} \sum_{q=1}^{2^{p}-1}(-1)^{m+q} 2^{-n-p} F\left(\frac{m k}{2^{n}}, \frac{q k}{2^{p}}\right) \\
& \times \cos \left(\frac{m x k}{2^{n}}\right) \cos \left(\frac{q y k}{2^{p}}\right) e^{i z k \sqrt{1-m^{2} / 4^{n}-q^{2} / 4^{p}}} .
\end{aligned}
$$

It can be clearly seen from (5.7) that the resulting field is due to a summation of an infinite number of plane waves, each propagating at a unique phase velocity (and hence a unique group velocity) based on its value of $k_{x}$ and $k_{y}$. Thus, the process of diffraction leads to a continuous distribution of group velocities, having an amplitude distribution governed by the Fourier transform of the aperture. This insight becomes particularly clear upon application of (2.4) to expand (5.2). Such a result has an effect on Doppler shifts both in acoustics and in electromagnetic wave propagation, effects that are not otherwise apparent without the use of the generalized method of exhaustion. 


\section{REFERENCES}

[1] M. Born and E. Wolf, Principles of Optics, 7th ed., Cambridge University Press, 1999.

[2] L. M. Brekhovskikh, Waves in Layered Media, Applied Mathematics and Mechanics, vol. 6, Academic Press, New York, 1960.

[3] G. C. Gaunaurd and H. Überall, Acoustics of finite beams, J. Acoust. Soc. Amer. 63 (1978), no. 1, 5-16.

[4] D. Grieser, private communication.

[5] A. A. Ruffa, A series for $\sin x / x$, Math. Mag. 73 (2000), no. 4, 321.

[6] G. F. Simmons, Calculus with Analytic Geometry, McGraw-Hill, New York, 1996.

Anthony A. Ruffa: Naval Undersea Warfare Center Division, 1176 Howell Street, NEWPORT, RI 02841-1708, USA 


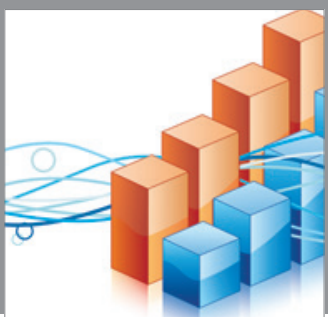

Advances in

Operations Research

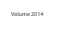

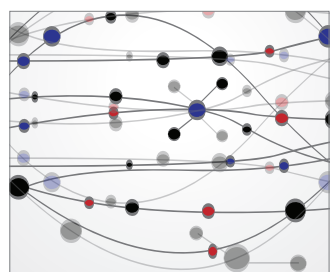

\section{The Scientific} World Journal
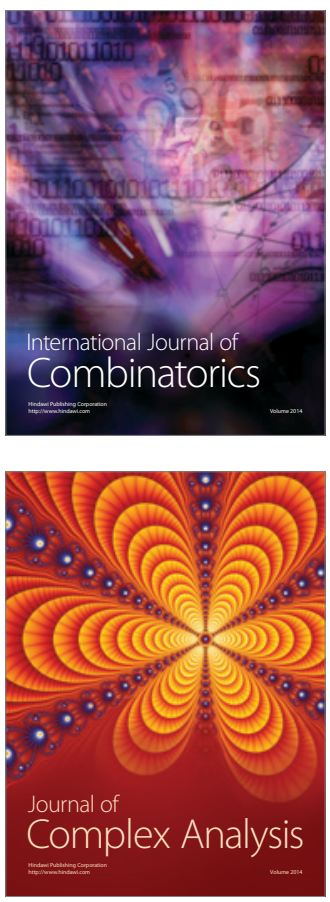

International Journal of

Mathematics and

Mathematical

Sciences
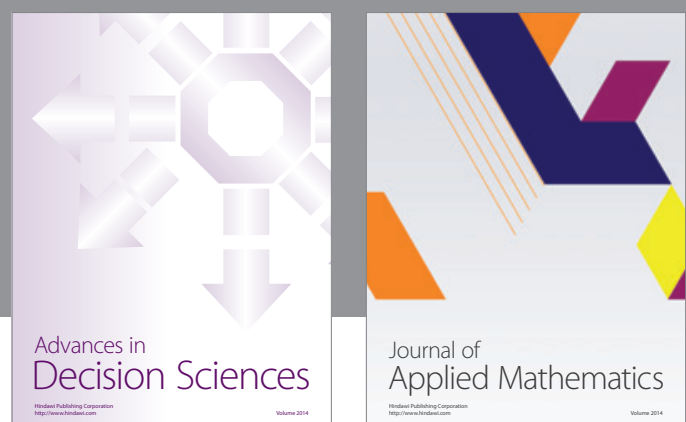

Journal of

Applied Mathematics
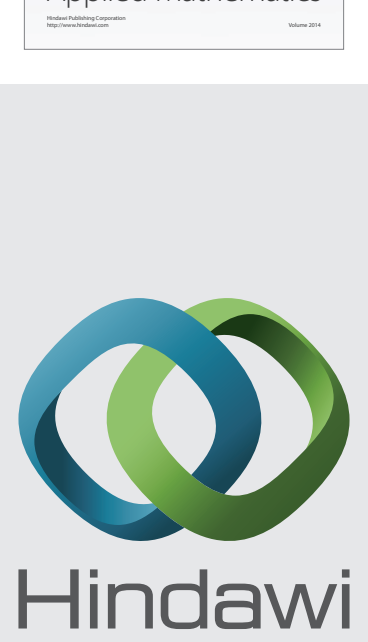

Submit your manuscripts at http://www.hindawi.com
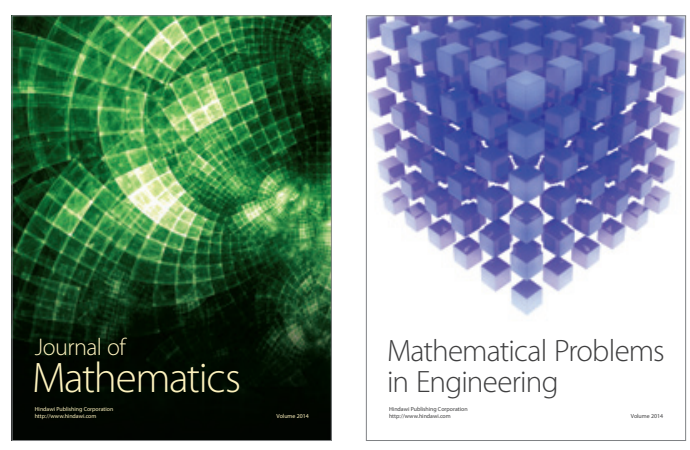

Mathematical Problems in Engineering
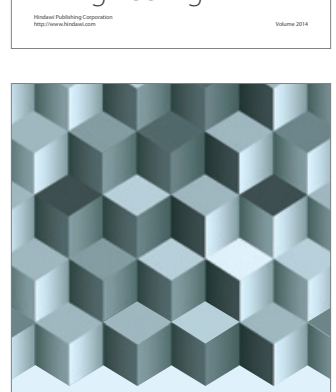

Journal of

Function Spaces
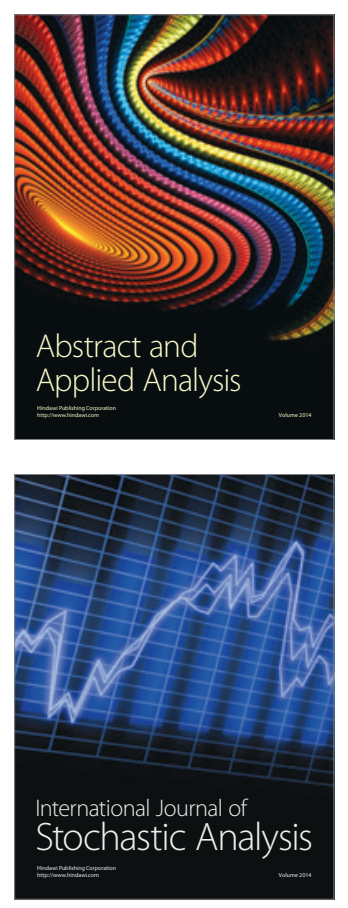

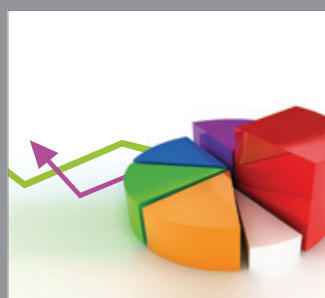

ournal of

Probability and Statistics

Promensencen
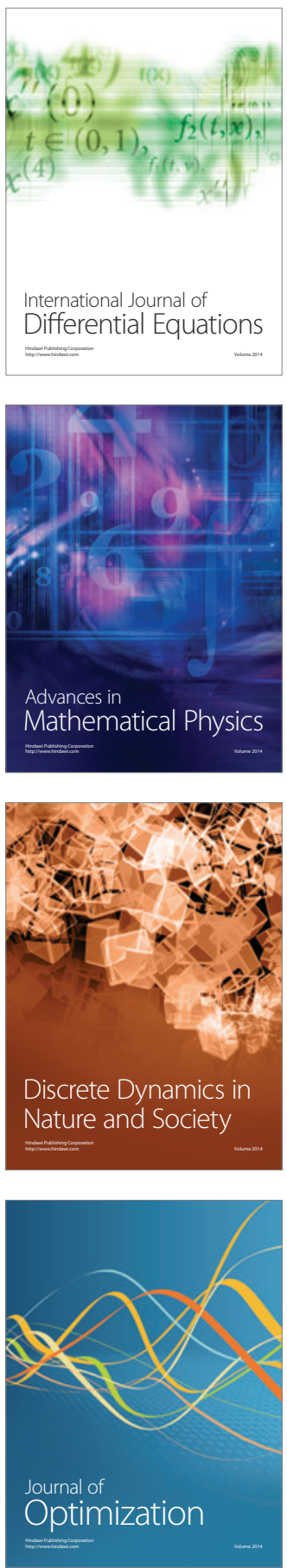